CLINICAL STUDY

\title{
Increased aortic root diameters in patients with acromegaly
}

Agatha A van der Klaauw ${ }^{1}$, Jeroen J Bax², Johannes W A Smit ${ }^{1}$, Eduard R Holman ${ }^{2}$, Victoria Delgado ${ }^{2}$, Gabe B Bleeker ${ }^{2}$, Nienke R Biermasz ${ }^{1}$, Ferdinand Roelfsema ${ }^{1}$, Johannes A Romijn ${ }^{1}$ and Alberto M Pereira ${ }^{1}$

Departments of ${ }^{1}$ Endocrinology and Metabolism and ${ }^{2}$ Cardiology, C4-R, Leiden University Medical Center, PO Box 9600,2300 RC Leiden, The Netherlands

(Correspondence should be addressed to A A van der Klaauw; Email: a.a.van_der_klaauw@lumc.nl)

\begin{abstract}
Objective: The clinical manifestations of acromegalic cardiomyopathy include arrhythmias, valvular regurgitation, concentric left ventricular (LV) hypertrophy, and LV systolic and diastolic dysfunction. At present, it is unknown whether acromegaly also affects the aortic root.

Design: Aortic root diameters were prospectively assessed in 37 acromegalic patients (18 patients with active disease and 19 with controlled disease) by conventional two-dimensional and Doppler echocardiography before, and after, an observation period of 1.9 years (range 1.5-3.0 years). Baseline parameters were compared with healthy controls.

Results: The diameters of the aortic root at the sino-tubular junction and the ascending aorta were increased in patients with acromegaly: $30 \pm 4 \mathrm{vs} 26 \pm 3 \mathrm{~mm}(P=0.0001)$ and $33 \pm 5 \mathrm{vs} 30 \pm 4 \mathrm{~mm}$ $(P=0.006)$ respectively. The diameter of the aortic root at the aortic annulus and aortic sinus were not different from controls. During follow-up, the aortic root diameters increased at the levels of the annulus and the sinotubular junction $(P=0.025$ and $P=0.024$ respectively), whereas there was no change in the diameters at the levels of the sinus and the ascending aorta during follow-up. Baseline aortic root diameters were not influenced by disease duration, current disease activity, or blood pressure. When patients with active and inactive disease were analyzed separately, only the diameter of the sinotubular junction increased in patients with inactive acromegaly during follow-up $(P=0.031)$.

Conclusion: Aortic root diameters are increased in patients with acromegaly compared with healthy controls.
\end{abstract}

European Journal of Endocrinology 159 97-103

\section{Introduction}

Acromegaly is associated with increased cardiovascular morbidity and mortality (1). Active disease leads to a specific acromegalic cardiomyopathy, which involves the myocardium, the conduction system, and the valves (1). Clinical manifestations include arrhythmias, valvular regurgitation, concentric, left ventricular (LV) hypertrophy, and LV systolic and diastolic dysfunction. Ten percent of the patients present with overt heart failure. Treatment of growth hormone $(\mathrm{GH})$ excess can reduce mortality to that of the normal population (2) and reverse heart failure and myocardial hypertrophy, but valvular regurgitation persists, or even aggravates, when disease activity is insufficiently controlled $(3,4)$. The pathophysiology of these cardiac complications of acromegaly is incompletely understood. On pathological examination, the myocardium is affected by interstitial fibrosis and the leaflets have the appearance of myxoid degeneration (5). It has been hypothesized that abnormal extracellular matrix regulation by overproduction of $\mathrm{GH}$ and/or insulin-like growth factor-I (IGF-I) in patients with acromegaly may contribute to both systolic and diastolic LV dysfunction. Abnormalities in matrix regulation are associated with cardiac chamber dilation and reduced myocardial tensile strength (6). Abnormal extracellular matrix regulation is also present in Marfan's syndrome and in this syndrome myxoid degeneration extends to the aortic root, which progressively dilates over time, with the need for surgical procedures to avoid dissection or severe aortic regurgitation (7).

It is currently unknown whether the aortic root is also involved in acromegaly, as in Marfan's syndrome. This is of clinical relevance for the appreciation of the extensiveness of the cardiac involvement in patients with acromegaly. Therefore, the aim of the present study was to compare the diameters of the aortic root between patients with acromegaly and healthy controls. In addition, we prospectively evaluated the effects of disease control on aortic root diameters during followup of 1.5-3 years.

\section{Patients and methods}

\section{Patients}

In the present study, 37 patients were enrolled, of whom 35 had participated in a previous cross-sectional study on 
cardiac function and valvular regurgitation in acromegaly, which did not involve assessment of cardiac root parameters (5). The initial diagnosis of acromegaly was based on the characteristic clinical signs and symptoms and confirmed by insufficient suppression of GH during a glucose tolerance test (normal response: GH nadir < $0.5 \mu \mathrm{g} / \mathrm{l}$ ), elevated age- and gender-adjusted IGF-I levels, and the presence of a pituitary adenoma on radiological imaging. The study was performed prior to the introduction of GH receptor blockade drugs in the Netherlands.

Patients were classified at study entry as having active or inactive acromegaly. Active acromegaly $(n=18)$ was defined as mean fasting GH concentrations (measured every $30 \mathrm{~min}$ for $3 \mathrm{~h}$ ) $>2.5 \mu \mathrm{g} / \mathrm{l}$ and elevated age- and gender-adjusted IGF-I concentrations. Nineteen patients were classified as having inactive acromegaly, which was defined in medically well-controlled patients $(n=13)$ as mean fasting $\mathrm{GH}$ concentration (measured for $3 \mathrm{~h}$ with an interval of $30 \mathrm{~min})<2.5 \mu \mathrm{g} / \mathrm{l}$ and normal age- and gender-adjusted IGF-I concentrations during treatment with somatostatin analogs $(n=13)$ and in cured acromegaly after surgery and radiotherapy $(n=6, n=5$ surgery alone; $n=1$ surgery and radiotherapy) as glucose-suppressed GH $<0.5 \mu \mathrm{g} / \mathrm{l}$ and normal age- and gender-adjusted IGF-I concentration without medical treatment. Of the 13 patients treated with somatostatin analogs, 6 were treated primarily by surgery and 2 with surgery and radiotherapy, whereas in 5 patients somatostatin analogs formed the primary treatment.

One patient presented with severe aortic regurgitation. This patient was excluded from the comparison with healthy controls of the diameter of the aortic root at the level of the aortic annulus and the follow-up data within patients with acromegaly, because the patient underwent valve replacement surgery.

Two male patients (aged 73 and 56 years respectively, active acromegaly throughout follow-up) were treated for diabetes mellitus. Neither had aortic regurgitation. Two patients used lipid lowering drugs (one male patient, age 73 years, active acromegaly throughout follow-up, without aortic regurgitation, and one female patient, age 63 years, cured acromegaly throughout follow-up, no aortic regurgitation). Two patients were using dopamine agonists at the time of the study. Neither had aortic regurgitation. None of the other patients used dopamine agonists.

None of the patients had hemodynamic instability, previous myocardial infarction, thyreotoxicosis, rheumatic fever, endocarditis, or connective tissue disease. The medical ethics committee of the Leiden University Medical Center approved the study, and written informed consent was obtained from all subjects.

\section{Controls}

The patients were compared with 37 healthy age-, body surface area (BSA)- and sex-matched controls. The controls were selected from a database with patients referred to the Department of Cardiology, based on age, sex, BSA, mitral and aortic valvular regurgitation, and LV systolic function. Controls were excluded when referred for echocardiographic evaluation of known valvular heart disease, murmur, congestive heart failure, and cardiac transplantation. Other exclusion criteria were myocardial infarction, thyreotoxicosis, rheumatic fever, endocarditis, connective tissue disease, carcinoid syndrome, or use of anorectic drugs. We and others have previously demonstrated that controls recruited from a large database can also be used as representative controls $(5,8)$.

\section{Echocardiography, data acquisition}

Aortic root diameters in patients with acromegaly were prospectively evaluated twice: at baseline and after followup duration of at least 1.5 years. Baseline parameters were compared with similar parameters in healthy controls. The evaluation of the echocardiographic images was performed blinded for the status of the subjects.

Echocardiography was performed while the patients were in the left lateral decubitus position using a commercially available system (Vingmed Vivid-7, General Electric - Vingmed, Milwaukee, WI, USA). Standard parasternal (long and short axes) and apical views (two, four, and five chambers) were obtained. M-mode images were obtained from the parasternal long-axis views for quantitative assessment of $\mathrm{LV}$ dimensions.

Standard continuous-wave and pulsed-wave Doppler examinations were performed. The severity of valvular regurgitation was assessed by two independent expert readers blinded to the clinical data on a qualitative scale of trace, mild, moderate, or severe, using previously described methods $(9,10)$. Significant valvular disease was determined using the US Food and Drug Administration case definition: mild or greater aortic regurgitation or mitral regurgitation equal to or more than moderate severity (11).

The aortic root was measured in the parasternal longaxis view at end diastole, perpendicular to the long axis of the aorta, according to the leading-edge technique at four levels: 1) annulus aortae, 2) sinuses of Valsalva, 3) sinotubular junction, and 4) proximal ascending aorta.

BSA was calculated by the formula proposed by Dubois et al. (12): $0.007184 \times$ weight $(\mathrm{kg}){ }^{0.425} \times$ length $(\mathrm{cm})^{0.725}$. Aortic root diameters were indexed for BSA as proposed by Roman et al. (13).

\section{Hormone assays}

GH concentrations were measured using a sensitive timeresolved immunofluorescent assay (Wallac Oy, Turku, Finland), specific for $22 \mathrm{kDa}$ GH protein. Human biosynthetic GH (Pharmacia and Upjohn, Inc.) was used as standard, calibrated against WHO-IRP 80-505. The detection limit was $0.012 \mu \mathrm{g} / \mathrm{l}$. Intra-assay coefficients of variation were $8.4-1.6 \%$ and the inter-assay coefficients of variation were interassay $9.0-2.0 \%$ in the GH-range 
$0.1-18 \mu \mathrm{g} / \mathrm{l}$. Total serum IGF-I concentration was determined by RIA after extraction and purification on ODS-silica columns (Incstar Corp., Stillwater, MN, USA). The intra- and inter-assay coefficients of variation were less than $11 \%$. The detection limit was $1.5 \mathrm{nmol} / \mathrm{l}$. Ageand gender-adjusted IGF-I data were determined in the same laboratory. IGF-I was expressed as a SDS from ageand gender-related normal levels.

\section{Statistical analysis}

Statistical analysis was performed using SPSS for Windows, version 14.0 (SPSS Inc., Chicago, IL, USA). Results are expressed as the mean \pm s.D., unless specified otherwise. Independent samples $t$-tests were used to assess the difference between patients and controls. Non-parametric Mann-Whitney $U$ tests and independent samples $t$-tests after log-transformation of the variables were used to check for consistency of the obtained results of the analysis. Paired samples $t$-tests were used to assess the difference in aortic root measurements at baseline and at follow-up. Independent samples $t$-tests or $\chi^{2}$ tests were used to compare baseline values, follow-up values, and the difference in baseline and follow-up values between patients with active and inactive acromegaly. Linear regression analysis was used to explore relations between aortic root diameters as dependent variables and variables of disease activity (IGF-I/GH concentration and estimated disease duration) and blood pressure as independent variables. A $P$ value $<0.05$ was considered to represent a significant difference.

\section{Results}

\section{Clinical characteristics}

Patients and controls were matched for age, gender, BSA, valvular regurgitation, and LV dimensions and function. The interval between the two echocardiographic studies in patients with acromegaly was 1.9 years, range 1.5-3.0 years. During follow-up, mean IGF-SDS, GH concentrations, and systolic blood pressure decreased (Table 1).

At baseline as well as at follow-up, mean IGF-I SDS were significantly higher in patients with active acromegaly compared with patients with inactive acromegaly $(8.3 \pm 1.9$ SDS versus $0.8 \pm 0.4$ SDS, $P<0.001$ at baseline, $2.9 \pm 0.5$ vs $1.0 \pm 0.3$, $P=0.006$ at follow-up respectively). During follow-up, all patients with active acromegaly were treated with somatostatin analogs. At the time of the first echocardiogram, no treatment had been started in nine of these patients. At the end of follow-up, disease activity in five of these patients was adequately controlled $(n=1$ after radiotherapy and somatostatin analogs, $n=1$ after surgery and somatostatin analogs and $n=3$ after somatostatin analogs alone). Disease activity was still classified as active in the other four patients despite surgery, radiotherapy, and somatostatin analogs $(n=1)$, or radiotherapy and somatostatin analogs $(n=1)$, or somatostatin analogs alone $(n=2)$. The other patients in the group of patients with active disease $(n=9)$ had been treated previously with surgery and somatostatin analogs $(n=4)$, somatostatin analogs $(n=4$ (one of these patients was classified as having adequate disease control at the end of follow-up)), and a combination of surgery, radiotherapy, and somatostatin analogs $(n=1)$. GH and IGF-I concentrations significantly decreased at the end of follow-up (GH: $19.0 \pm 4.9$ to $5.8 \pm 1.4 \mu \mathrm{g} / \mathrm{l}$, $P=0.017$ and IGF-1: $8.3 \pm 1.9$ to $2.9 \pm 0.5$ SDS, $P<0.001$ respectively). Disease activity in 6 of the 18 patients with active acromegaly at baseline was thus adequately controlled at the end of follow-up. The mean duration of adequate control of acromegaly in patients with inactive disease at study entry was 7.6 years (range 0.8-22.7 years). Mean estimated disease duration prior to baseline evaluation was 11 years (range 1-46 years).

Mean systolic blood decreased during follow-up from $144 \pm 23 \mathrm{mmHg}$ at baseline to $134 \pm 14 \mathrm{mmHg}$ at

Table 1 Clinical characteristics of patients with acromegaly compared with healthy age-, sex-, and body suface area-matched controls.

\begin{tabular}{|c|c|c|c|}
\hline & Acromegaly $(n=37)$ & Controls $(n=37)$ & $P$ value \\
\hline Age (years) & $54 \pm 14$ & $52 \pm 9$ & NS \\
\hline Gender (male/female $(n)$ ) & $15 / 22$ & $15 \overline{22}$ & NS \\
\hline Body surface area $\left(\mathrm{m}^{2}\right)$ & $2.0+0.2$ & $1.9+0.1$ & NS \\
\hline IGF-I at baseline (SDS) & $4.4 \pm 5.3$ & NA & \\
\hline IGF-I at follow-up (SDS) & $1.9 \pm 2.0^{*}$ & NA & \\
\hline $\mathrm{GH}$ at baseline $(\mu \mathrm{g} / \mathrm{l})$ & $10.7 \pm 16.9$ & NA & \\
\hline $\mathrm{GH}$ at follow-up $(\mu \mathrm{g} / \mathrm{l})$ & $3.8 \pm 4.6^{*}$ & NA & \\
\hline Left ventricular end diastolic diameter $(\mathrm{mm})$ & $51+7$ & $51+6$ & NS \\
\hline Left ventricular end systolic diameter ( $\mathrm{mm})$ & $34 \pm 6$ & $31 \pm 6$ & 0.07 \\
\hline Left ventricular ejection fraction (\%) & $67 \pm 11$ & $69 \pm 11$ & NS \\
\hline $\begin{array}{l}\text { Mitral valve regurgitation ( } n(\%) \text { no/trace/mild/moderate/ } \\
\text { severe regurgitation) }\end{array}$ & $25(68) / 8(22) / 4(10) / 0 / 0$ & $27(73) / 8(22) / 2(5) / 0 / 0$ & NS \\
\hline $\begin{array}{l}\text { Aortic valve regurgitation ( } n(\%) \text { no/trace/mild/moderate/ } \\
\text { severe regurgitation) }\end{array}$ & $27(73) / 4(11) / 5(14) / 0 / 1(2)$ & $33(90) / 2(5) / 2(5) / 0 / 0$ & NS \\
\hline
\end{tabular}

Values are expressed as mean \pm s.D. The two groups are compared with independent samples $t$-tests or $\chi^{2}$ tests where appropriate. ${ }^{*} P<0.05$ compared with baseline value of same parameter. 
follow-up $(P=0.002)$, whereas diastolic blood pressured remained unchanged $(84 \pm 8 \mathrm{mmHg}$ at baseline vs $84 \pm 9 \mathrm{mmHg}$ at follow-up, $P=0.0419$ ).

\section{Comparison with controls}

Compared with age-, sex-, and BSA-matched controls with similar LV function and valvular regurgitation, the diameters of the aortic root at the sino-tubular junction and the ascending aorta were increased in patients with acromegaly (Table 2 and Figure 1). However, the diameter of the aortic root at the aortic sinus and the aortic annulus were not different between patients and controls.

The diameter of the aortic root at the sino-tubular junction and the ascending aorta were increased in patients with acromegaly compared with controls also after exclusion of patients and controls with significant aortic regurgitation (more than trace, Table 3). The diameter of the aortic root at the aortic annulus and the aortic sinus was not different between these two latter groups. Secondly, we excluded all patients and controls with at least trace aortic regurgitation. This analysis again showed an increased aortic root diameter at the level of the sino-tubular junction and the ascending aorta in patients compared with controls ( $30 \pm 4$ vs $26 \pm 3 \mathrm{~mm}, P<0.001$ and $33 \pm 4$ vs $30 \pm 4, P=0.027$ respectively).

\section{Follow-up of all patients with acromegaly}

During follow-up of all patients with acromegaly, the diameter of the aortic root at the annulus and at the sinotubular junction increased (Table 4). However, the diameters at the level of the sinus and the ascending aorta did not differ between baseline and follow-up.

\section{Factors influencing aortic root diameters in patients with acromegaly}

Disease activity at baseline Baseline and follow-up diameters of the aortic root were not different between patients with active and inactive acromegaly. When

Table 2 Aortic root measurements in patients with acromegaly $(n=37)$ compared with healthy controls $(n=37)$.

\begin{tabular}{llll}
\hline & $\begin{array}{l}\text { Acromegaly } \\
(n=37)\end{array}$ & $\begin{array}{l}\text { Healthy } \\
\text { controls } \\
(n=37)\end{array}$ & $\boldsymbol{P}$ value \\
\hline Aortic annulus $(\mathrm{mm})^{\mathrm{a}}$ & $22 \pm 2$ & $21 \pm 2$ & $\mathrm{NS}$ \\
Sinuses of Valsalva (mm) & $32 \pm 4$ & $33 \pm 4$ & $\mathrm{NS}$ \\
$\begin{array}{l}\text { Sinotubular junction (mm) } \\
\text { Proximal ascending aorta }\end{array}$ & $30 \pm 4$ & $26 \pm 3$ & 0.0001 \\
$\quad(\mathrm{~mm})$ & & $30 \pm 4$ & 0.006 \\
\hline
\end{tabular}

Values are expressed as mean \pm s.D. Parameters between patients and controls are compared with independent samples $t$-tests.

${ }^{a}$ One patient presented with severe aortic regurgitation. This patient was excluded from the comparison with healthy controls of the diameter of the aortic root at the level of the aortic annulus.
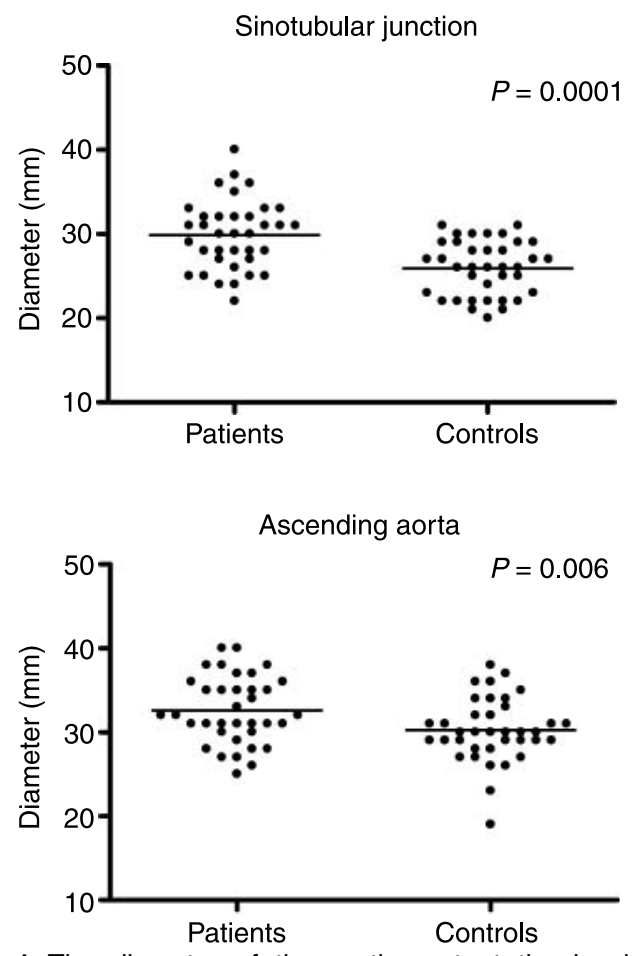

Figure 1 The diameter of the aortic root at the level of the sinotubular junction and the ascending aorta in patients with acromegaly compared with controls matched for age, gender, body surface area, and aortic regurgitation.

corrected for BSA or annular size, the ratios of sinuses of Valsalva and sinotubular junction were not different between patients with active and inactive disease. When patients with active and inactive disease were analyzed separately, only the diameter of the sinotubular junction increased in patients with inactive acromegaly $(P=0.031)$ (Table 5).

Estimated disease duration at baseline No correlations could be detected between estimated disease duration at baseline and any of the aortic root diameters (baseline or follow-up) or the difference between baseline and follow-up.

Table 3 Aortic root measurements in patients with acromegaly $(n=31)$ compared with healthy controls $(n=35)$ after exclusion of the subjects with significant aortic regurgitation (more than trace).

\begin{tabular}{lllc}
\hline & $\begin{array}{l}\text { Acromegaly } \\
(n=31)\end{array}$ & $\begin{array}{l}\text { Healthy } \\
\text { controls } \\
(n=35)\end{array}$ & P value \\
\hline Aortic annulus (mm) & $22 \pm 2$ & $21 \pm 2$ & NS \\
Sinuses of Valsalva (mm) & $32 \pm 4$ & $33 \pm 4$ & NS \\
Sinotubular junction (mm) & $30 \pm 4$ & $26 \pm 3$ & $<0.001$ \\
$\begin{array}{l}\text { Proximal ascending aorta } \\
(m m)\end{array}$ & $32 \pm 4$ & $30 \pm 4$ & 0.025 \\
\hline
\end{tabular}

Values are expressed as mean \pm S.D. Parameters between patients and controls are compared with independent samples $t$-tests. 
Table 4 Aortic root measurements in patients with acromegaly $(n=37)$ at baseline and during follow-up of 1.9 years (range 1.5-3.0 years).

\begin{tabular}{llll}
\hline & Baseline & Follow-up & $\boldsymbol{P}$ value \\
\hline Aortic annulus $(\mathrm{mm})$ & $22 \pm 2$ & $23 \pm 3$ & 0.025 \\
Sinuses of Valsalva $(\mathrm{mm})$ & $32 \pm 4$ & $33 \pm 3$ & NS \\
Sinotubular junction $(\mathrm{mm})$ & $30 \pm 4$ & $32 \pm 4$ & 0.024 \\
$\begin{array}{c}\text { Proximal ascending aorta } \\
\quad(\mathrm{mm})\end{array}$ & $33 \pm 4$ & $33 \pm 4$ & $\mathrm{NS}$ \\
& & &
\end{tabular}

Values are expressed as mean \pm s.D. Baseline and follow-up parameters are compared with paired samples $t$-tests.

Blood pressure No correlations were found between systolic and diastolic blood pressure and aortic root diameters at baseline or follow-up. The aortic root diameters were similar in patients with or without hypertension (systolic blood pressure $\geq 140 \mathrm{mmHg}$ and/or diastolic blood pressure $\geq 90 \mathrm{mmHg}, \quad n=14$ and $n=23$ respectively). Aortic annulus: $22 \pm 2$ vs $22 \pm 2 \mathrm{~mm}$ in patients with and without hypertension, $P=\mathrm{NS}$; Sinus: $33 \pm 5$ vs $32 \pm 4 \mathrm{~mm}, P=\mathrm{NS}$; Sinotubular junction: $30 \pm 4$ vs $30 \pm 4 \mathrm{~mm}, P=\mathrm{NS}$; Ascending aorta: $32 \pm 4$ vs $34 \pm 4 \mathrm{~mm}, P=\mathrm{NS}$ ).

Gender, weight, height, and body mass index (BMI) Diameters of the aortic root at all levels were higher in men than in women (data not shown) as were height and BSA. Weight and height were not correlated with diameters of the aortic root. BMI was positively correlated with the diameter at the aortic sinus $(R=$ $0.377, P=0.022$ ).

$\boldsymbol{L}$ function and dimensions There were no correlations between aortic diameters and LV ejection fraction/fractional shortening/end diastolic diameter or end systolic diameter. Diameters at the level of the aortic annulus and the ascending aorta were higher in patients with LV hypertrophy $(n=9,24 \%)$ compared with patients without $(22 \pm 2$ vs $24 \pm 3 \mathrm{~mm}, P=0.043$ respectively and $32 \pm 4$ vs $35 \pm 4 \mathrm{~mm}, P=0.051$ respectively). No differences were found in the diameters at the level of the sinus and sinotubular junction.

\section{Discussion}

This case-control and follow-up study evaluated in detail the diameters of the aortic root in patients with acromegaly. In general, the aortic root diameters were increased in patients with acromegaly compared with healthy controls. Disease activity or disease duration did not influence absolute aortic root diameters at baseline. During follow-up, the aortic root diameter at the aortic annulus and at the sinotubular junction increased in patients with acromegaly, irrespective of disease activity. These data indicate that the long-term exposure to $\mathrm{GH}$ excess in patients with acromegaly affects the aortic root in addition to previously documented effects on the aortic valve leaflets $(5,14,15)$.

The effects of acromegaly on the diameter of the aortic root have hardly been evaluated. To our knowledge, only one study assessed aortic root diameters in a limited number of patients, and aortic root enlargement was found in 1 out of 25 patients with active and inactive acromegaly (16). In the present study, we found increased diameters of the aortic root at several levels in patients with acromegaly compared with healthy controls. In the Framingham heart study, determinants of aortic root size were age, height, weight, and sex (17). Therefore, we matched our patients with healthy controls for these factors. To avoid other confounders in this specific comparison, patients and controls were also matched for LV function and valvular regurgitation. The additional influence of increases in blood pressures is limited (17). Accordingly, blood pressure did not influence aortic root diameters or dilatation during follow-up in our study. Moreover, during follow-up, systolic blood pressure decreased due to more stringent disease control in the majority of patients with active disease, illustrated by the decrease in GH and IGF-I SDS. $\mathrm{GH}$ has direct effects on potassium reabsorption, hence increasing the extracellular volume. Nonetheless, the diameter of the aortic root increased at the level of the

Table 5 Aortic root diameters and disease activity at baseline and during follow-up in patients with active versus inactive acromegaly.

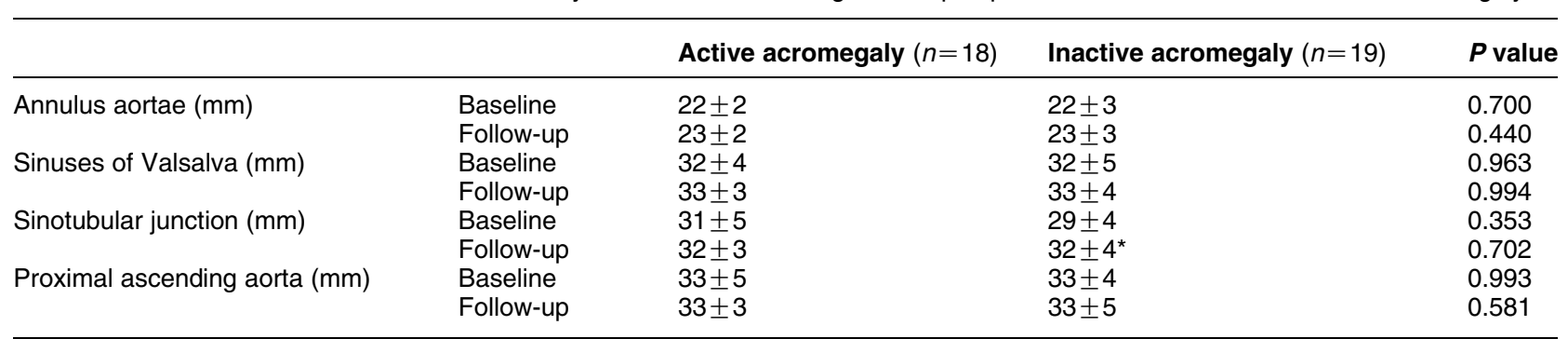

Values are expressed as mean \pm s.D. Within the two groups, parameters are compared between baseline and follow-up with paired samples $t$-tests. The two groups are compared with independent samples $t$-tests. ${ }^{\star} P=0.031$ compared with baseline in patients with inactive acromegaly. 
aortic annulus and the sinotubular junction during follow-up. We feel that this increase in aortic root diameters despite lowering of blood pressure, suggests that blood pressure is not a major determinant of aortic root diameters in this particular patient group. It is, therefore, unlikely that uncontrolled hypertension influenced our results.

There was no correlation between dilation of the aortic root and current disease activity or estimated disease duration. Although a similar lack of association was found between valvular regurgitation and current disease activity, valvular regurgitation was strongly associated with disease duration (5), pointing towards direct effects of long-term exposure to increased $\mathrm{GH}$ and/or IGF-1 concentrations on cardiac valves. These observations indicate that the detrimental effects apparently only become manifest after long-term exposure, and at best the reversibility is only partial. However, to evaluate the effects of current disease state on progressive abnormalities of the aortic root, a study with a longer follow-up duration and with more patients is needed.

We speculated that the increased diameters of the aortic root are probably due to the same mechanisms that induce the myxomatous degeneration found in the valves that were removed from several of our acromegalic patients during valvular replacement surgery (5). GH is involved in matrix regulation. For example GH increases gene expression of the matrix metalloproteinases, which are capable of altering the composition of the extracellular matrix (18). This altered matrix regulation could be responsible for the changes found in the heart valves, as well as in the aortic root in patients with acromegaly. The coincidence between valvular regurgitation and aortic root dilatation is also present in Marfan's syndrome, which is also characterized by myxomatous degeneration of cardiac valves and aortic root (7).

We found an increase in the diameter of the aortic root at the sinotubular junction in patients with inactive acromegaly that is somewhat unexpected. The diameter of the aortic root also increased in patients with active acromegaly, although this did not reach statistical significance, and when all patients are analyzed together. These data might suggest that the long-standing GH excess has altered the aortic root in such a way that it is more vulnerable to distension even when GH excess is fully reversed.

None of our patients were diagnosed with true thoracic aortic aneurysms. Therefore, the results of the present study do not imply that aortic root diameters should be screened in all patients with acromegaly to detect aneurysms. However, extending the echocardiographic measurements to the aortic root offers a more complete picture of the spectrum of acromegalic cardiomyopathy.

In conclusion, aortic root diameters were increased in patients with acromegaly compared with healthy controls. These abnormalities were not associated with disease duration, current disease activity, or blood pressure. In addition, during follow-up, aortic root diameters at the level of the aortic annulus and the sinotubular junction increased. These findings indicate that in patients with acromegaly an extension of the cardiac evaluation to the aortic root offers a more indebt assessment of the state of the individual acromegalic cardiomyopathy.

\section{Acknowledgements}

A A van der Klaauw is supported by an AGIKO grant of the Netherlands Organisation for Health Research and Development (grant number: 92003423).

\section{References}

1 Colao A, Ferone D, Marzullo P \& Lombardi G. Systemic complications of acromegaly: epidemiology, pathogenesis, and management. Endocrine Reviews 200425 102-152.

2 Biermasz NR, Dekker FW, Pereira AM, van Thiel SW, Schutte PJ, van Dulken H, Romijn JA \& Roelfsema F. Determinants of survival in treated acromegaly in a single center: predictive value of serial insulin-like growth factor I measurements. Journal of Clinical Endocrinology and Metabolism 200489 2789-2796.

3 Colao A, Marzullo P, Ferone D, Spinelli L, Cuocolo A, Bonaduce D, Salvatore M, Boerlin V, Lancranjan I \& Lombardi G. Cardiovascular effects of depot long-acting somatostatin analog Sandostatin LAR in acromegaly. Journal of Clinical Endocrinology and Metabolism $2000853132-3140$.

4 Colao A, Cuocolo A, Marzullo P, Nicolai E, Ferone D, Della Morte AM, Pivonello R, Salvatore M \& Lombardi G. Is the acromegalic cardiomyopathy reversible? Effect of 5-year normalization of growth hormone and insulin-like growth factor I levels on cardiac performance Journal of Clinical Endocrinology and Metabolism 200186 1551-1557.

5 Pereira AM, van Thiel SW, Lindner JR, Roelfsema F, van der Wall EE, Morreau H, Smit JW, Romijn JA \& Bax JJ. Increased prevalence of regurgitant valvular heart disease in acromegaly. Journal of Clinical Endocrinology and Metabolism 2004 89 71-75.

6 Miner EC \& Miller WL. A look between the cardiomyocytes: the extracellular matrix in heart failure. Mayo Clinic Proceedings 2006 $8171-76$.

7 van Karnebeek CD, Naeff MS, Mulder BJ, Hennekam RC \& Offringa M. Natural history of cardiovascular manifestations in Marfan syndrome. Archives of Disease in Childhood 2001 84 129-137.

8 Zanettini R, Antonini A, Gatto G, Gentile R, Tesei S \& Pezzoli G. Valvular heart disease and the use of dopamine agonists for Parkinson's disease. New England Journal of Medicine 2007356 39-46.

9 Perry GJ, Helmcke F, Nanda NC, Byard C \& Soto B. Evaluation of aortic insufficiency by Doppler color flow mapping. Journal of the American College of Cardiology $19879952-959$.

10 Thomas JD. How leaky is that mitral valve? Simplified Doppler methods to measure regurgitant orifice area Circulation 199795 548-550.

11 Weissman NJ, Tighe JF Jr, Gottdiener JS \& Gwynne JT. An assessment of heart-valve abnormalities in obese patients taking dexfenfluramine, sustained-release dexfenfluramine, or placebo. Sustained-Release Dexfenfluramine Study Group. New England Journal of Medicine 1998339 725-732.

12 Dubois D \& Dubois EF. A formula to estimate the approximate surface area if height and weight be known. Archives of Internal Medicine, 1916 863-871. 
13 Roman MJ, Devereux RB, Kramer-Fox R \& O'Loughlin J. Two-dimensional echocardiographic aortic root dimensions in normal children and adults. American Journal of Cardiology 1989 64 507-512.

14 Colao A, Spinelli L, Marzullo P, Pivonello R, Petretta M, Di Somma C, Vitale G, Bonaduce D \& Lombardi G. High prevalence of cardiac valve disease in acromegaly: an observational, analytical, case-control study. Journal of Clinical Endocrinology and Metabolism 200388 3196-3201.

15 van der Klaauw AA, Bax JJ, Roelfsema F, Bleeker GB, Holman ER, Corssmit EP, van der Wall EE, Smit JW, Romijn JA \& Pereira AM. Uncontrolled acromegaly is associated with progressive mitral valvular regurgitation. Growth Hormone and IGF Research 2006 16 101-107.
16 Savage DD, Henry WL, Eastman RC, Borer JS \& Gorden P. Echocardiographic assessment of cardiac anatomy and function in acromegalic patients. American Journal of Medicine 197967 823-829.

17 Vasan RS, Larson MG \& Levy D. Determinants of echocardiographic aortic root size. The Framingham Heart Study. Circulation $199591734-740$.

18 Thompson BJ, Shang CA \& Waters MJ. Identification of genes induced by growth hormone in rat liver using cDNA arrays. Endocrinology $2000 \mathbf{1 4 1} 4321-4324$.

Received 29 April 2008

Accepted 19 May 2008 